\title{
Liside pitagorico, sepolto a Tebe
}

Lysis pythagoricien, enseveli à Thèbes

Lysis of Taras, Disciple of Pythagoras, Buried in Thebes

\section{Federica Cordano}

\section{(2) OpenEdition}

\section{Journals}

Edizione digitale

URL: https://journals.openedition.org/gaia/415

DOI: 10.4000/gaia.415

ISSN: $2275-4776$

\section{Editore}

UGA Éditions/Université Grenoble Alpes

\section{Edizione cartacea}

ISBN: 978-2-37747-057-0

ISSN: 1287-3349

\section{Notizia bibliografica digitale}

Federica Cordano, «Liside pitagorico, sepolto a Tebe», Gaia [Online], 21 | 2018, online dal 01 novembre 2018, consultato il 10 décembre 2021. URL: http://journals.openedition.org/gaia/415; DOI: https:// doi.org/10.4000/gaia.415

Questo documento è stato generato automaticamente il 10 décembre 2021.

Gaia. Revue interdisciplinaire sur la Grèce archaïque 


\title{
Liside pitagorico, sepolto a Tebe
}

\author{
Lysis pythagoricien, enseveli à Thèbes \\ Lysis of Taras, Disciple of Pythagoras, Buried in Thebes
}

Federica Cordano

\section{Sepolture di pitagorici}

1 Nel de genio Socratis plutarcheo (Mor. 585-586) ${ }^{1}$, il pitagorico Teanore spiega a Simmia ${ }^{2}$ come e perché Liside di Taranto ${ }^{3}$ abbia ricevuto a Tebe la sepoltura giusta per un pitagorico e come questo sia avvenuto per merito di Epaminonda.

2 Teanore 4 è giunto a Tebe dall'Italia, dopo aver appreso in sogno ${ }^{5}$ della morte di Liside, per verificare come era stato sepolto, dal momento che il luttuoso evento era accaduto in terra straniera, e pronto, se vi avesse trovato dei difetti, a trasferire la salma in Italia

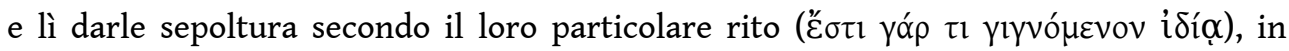

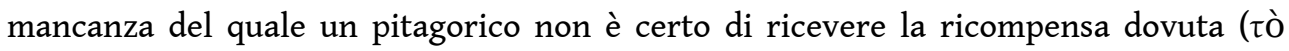

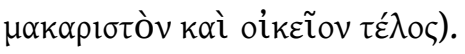

3 La traslazione non è poi avvenuta perché Teanore ha sentito una voce che lo rassicurava sulla sepoltura di Liside, eseguita secondo il "sacro rito" ed aggiungeva che

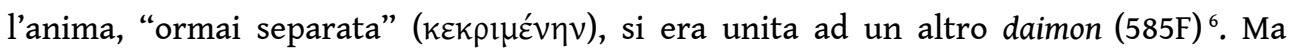
ancor prima, Teanore aveva saputo dallo stesso Epaminonda come era avvenuta la sepoltura e, quindi, aveva capito «che era stato bene istruito da quell'uomo fino alla conoscenza dei misteri ( $\tau \grave{\alpha} \alpha \dot{\alpha} \pi \circ \rho \rho \eta ́ \tau \alpha)^{7} »$.

Giamblico (V.P., 85) ci dice che erano stabilite le modalità «nelle quali vanno celebrati, nelle diverse circostanze, i riti sacrificali, in particolare quelli relativi alla dipartita

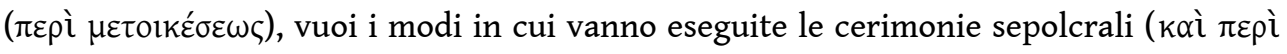
$\tau \alpha \grave{\Lambda} \varsigma \tau \alpha \varphi \alpha \varsigma \pi \tilde{\omega} \varsigma \delta \varepsilon \tilde{\varepsilon} \kappa \alpha \tau \alpha \theta \alpha \pi \tau \dot{\varepsilon} \sigma \theta \alpha 1) »^{8}$, noi non sappiamo quali fossero, sappiamo però qualche altra cosa sulla preoccupazione dei pitagorici al riguardo.

5 Una delle varie spiegazioni della salvezza di Pitagora al momento della strage da parte dei Ciloniani, è la notizia che egli era andato a Delo per assistere Ferecide di Siro nella malattia e per seppellirlo'; la notizia si trova in Diodoro (X, 11), in Giamblico (V.P., $248 \mathrm{e}$ 
252) da Nicomaco di Gerasa ${ }^{10}$, in Porfirio (V.P., 15 e 55) e anche in Diogene Laerzio (I, 118 e VIII, 40), che attinge ad Aristosseno ${ }^{11}$ e ad Eraclide Lembo ${ }^{12}$, il quale aggiungeva che Pitagora, tornando da Delo, andò a Metaponto e lì morì di digiuno.

6 Sul luogo della morte - e si presume di sepoltura - di Pitagora a Metaponto, le fonti non sono chiarissime e tanto meno la moderna interpretazione di esse. Pitagora è morto, secondo Porfirio, nel santuario delle Muse, secondo altri nella sua casa: è del tutto probabile che siano la stessa cosa e che lo «stenopos delle Muse » sia l'indirizzo di Pitagora a Metaponto ${ }^{13}$; come è altrettanto esplicito nella stessa tradizione che la casa di Pitagora a Metaponto, o quella che era ritenuta tale, divenne il santuario di Demetra.

7 Timeo citato da Porfirio (V.P., 4$)^{14}$, spostava tutto questo racconto a Crotone, forse per errore dello stesso Porfirio, non intendo fermarmi su questo ma sottolineare invece il rapporto con Demetra, la dea che conosce la strada degli inferi, percorsa da Pitagora in vita e certamente dalla sua psyche alla ricerca di un altro daimon (de genio S., 585F $)^{15}$.

8 Mi pare utile ricordare, soprattutto in questa sede, che un'altra casa importante, quella di Cadmo, divenne un santuario di Demetra (Paus., IX, 16,5) ${ }^{16}$; a Tebe, però l'associazione fra l'eroe e la divinità ha una valenza politica che a Metaponto (Crotone?) si era persa con il tempo.

9 Altre tradizioni, seppur limitate e reticenti, ci dicono qualcosa sulle sepolture dei Pitagorici ${ }^{17}$; la più antica è un famoso passo di Erodoto (II, 81$)^{18}$ nel quale si afferma che essi, come gli Egiziani, avvolgevano il cadavere in panni di colore bianco ${ }^{19}$, questo uso presuppone l'inumazione del cadavere, pratica che è espressamente imposta in Giamblico (V.P., 154): «non permetteva che, alla maniera dei Magi, si cremassero i cadaveri, perché voleva evitare che la materia mortale contaminasse un elemento

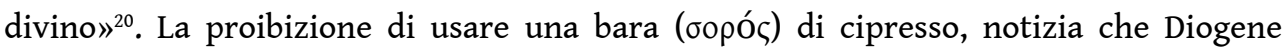
Laerzio (VIII, 10) attribuisce ad Ermippo ${ }^{21}$, presuppone la deposizione di un corpo inumato.

10 Di contenitori fittili, ornati di foglie di mirto, di ulivo e di pioppo, ci parla Plinio il Vecchio (XXXV, 60) quando descrive la sepoltura di Varrone, avvenuta secondo gli usi pitagorici: «quin et defunctos sese multi fictilibus soliis condi maluere sicut M. Varro, pythagorico modo in myrti et oleae atque populi nigrae foliis».

11 In aperto contrasto con questa tradizione è quanto si legge in Valerio Massimo (VII, 1,4): «Cuius ardentem rogum plenis venerationis oculis Metapontum aspexit oppidum, Pythagorae quam suorum cinerum nobilius clariusque monumentum».

12 Certamente secondo le regole della Scuola era stato inumato ${ }^{22}$ nel I sec. a.C., ad Alasehir, presso Philadelpheia di Lidia ${ }^{23}$, un discepolo di nome Pitagora, che aveva scelto la via del $\pi$ óvoৎ, come si legge nell'epigramma inciso nella parte inferiore della stele di marmo. Esso era originariamente composto da quattro pentametri, ma le sei lettere superstiti del quarto pentametro non ne permettono l'integrazione.

13 La lapide è decorata con simboli neopitagorici, che però riconducono facilmente ad una antica e reiterata tradizione - si pensi ad Esiodo ${ }^{24}$ e Prodico di Ceo citati da

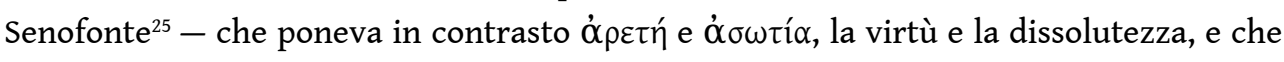
premiava il $\pi$ óvo sopportato in vita dall'uomo virtuoso ${ }^{26}$.

14 La stele è divisa in cinque settori dalla $Y$, simbolo del bivio fra $\mathrm{i}$ due opposti comportamenti umani; nei campi in basso sono rappresentate in veste femminile e nominate con iscrizioni, $\dot{\alpha} \rho \varepsilon \tau \eta ́$ e $\dot{\alpha} \sigma \omega \tau i ́ \alpha$, entrambe accompagnate da un bambino; in 
quelli in alto è raffigurato, a destra, l'uomo che lavora e che gode del premio; a sinistra l'uomo debosciato e quindi castigato, al centro, forse, il defunto.

F. Cumont, in un libro memorabile ${ }^{27}$, ha fatto memoria di altri monumenti funerari con il motivo della $Y(\mathrm{e} \text { anche della } \Psi)^{28}$, ma la più sorprendente affinità con il monumento di Alasehir si trova nella cosiddetta Tabula Cebetis ${ }^{29}$, un testo di autore anonimo che ha scelto, non a caso, il nome di Cebete tebano, socratico ma anche pitagorico, per illustrare la situazione dell'adolescente che si trova al bivio della vita. A. Carlini giustamente trovava in questa affinità la prova che il testo della Tabula è nato in un ambiente pitagorico del I sec. d.c. ${ }^{30}$.

\section{Liside ed Epaminonda}

«Dei due che sopravvissero, entrambi tarantini, Archippo si ritirò nella sua città, mentre Liside, presa a detestare la generale indifferenza, partì per la Grecia, visse nell'Acaia peloponnesiaca e poi si trasferì a Tebe, dove trovò un certo seguito. Qui divenne suo discepolo Epaminonda, che giunse a chiamarlo padre, e qui infine morì»: queste le parole di Giamblico (V.P., 250, p. 499) nella sapiente traduzione di Maurizio Giangiulio; esse pongono naturalmente dei problemi, che non vengono risolti dal confronto con le altre fonti utili al tema. Lo stesso Giangiulio, nel commento al passo, chiarisce che i moti pitagorici cui si fa qui riferimento sono ravvisabili negli anni intorno alla metà del V sec. a.C., e soprattutto prima della alleanza di Crotone con la Thurii democratica (Diod., XII, 11,3), che non sarebbe stata mai stipulata da Crotoniati in prevalenza pitagorici ${ }^{31}$.

17 Nel de genio Socratis plutarcheo, il disastroso incendio di una casa piena di pitagorici si svolge a Metaponto, anziché a Crotone, ed i giovani superstiti sono Liside e Filolao $(583 \mathrm{a})^{32}$. Però qui Filolao trova rifugio tra i Lucani, in corrispondenza con la tradizione polibiana che enfatizza l'espansione dei circoli pitagorici in Italia ${ }^{33}$. È l'opera nella quale è rappresentata al meglio la persistenza della dottrina pitagorica nelle città della Grecia continentale, sopra tutte Fliunte e Tebe, città nelle quali gli esiliati mantengono viva la tradizione pitagorica, senza interrompere il rapporto con l'Italia, come ci narra la storia della delegazione capeggiata da Teanore.

18 La tradizione sulla permanenza di Liside a Tebe poggia, naturalmente, sul suo rapporto con Epaminonda. Le fonti sottolineano la vecchiezza di Liside e la giovinezza di Epaminonda per accostare il più possibile la vita dei protagonisti, però non si può sospettare del rapporto intercorso fra i due, che può risalire al padre di Epaminonda, che era della stessa generazione di Liside.

19 Liside è certamente stato attirato a Tebe dalla presenza di una preesistente scuola pitagorica, frequentata da Filolao ${ }^{34} \mathrm{e}$ Cebete Tebano trasferendosi lì dalla prima sede di esilio in Acaia, e quella scuola continuerà dopo la morte di Liside, come ci suggerisce la notizia che il giovane Filippo figlio di Aminta ne apprese la dottrina nel suo esilio tebano, certamente successivo alla morte di Liside ${ }^{35}$.

Cornelio Nepote inizia la sua biografia di Epaminonda sottolineandone la povertà e la grande cultura: per la 'filosofia' egli ebbe come maestro Liside di Taranto, Pitagorico, quando era adulescens, poi, da ephebus cominciò la palestra, dove preferiva la corsa e la lotta, e, aggiunge Nepote, si dedicava con particolare attenzione alle $\mathrm{armi}^{36}$. Il momento del passaggio dall'adolescenza all'efebia è proprio quello nel quale si trova Eracle al 


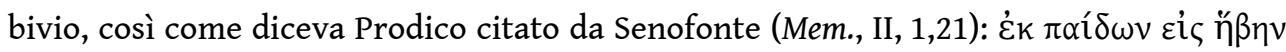
$\dot{\omega} \rho \mu \tilde{\alpha} \tau$ !

21 In Pausania (IX, 13) ritroviamo le due affermazioni di Nepote sulla modesta fortuna della famiglia di Epaminonda e sulla sua frequentazione di Liside Tarantino, quando era

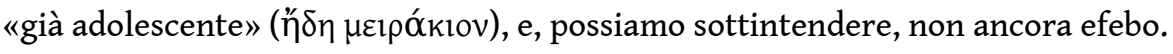

Nel decimo libro di Diodoro Siculo (c.11) questo rapporto tra un maestro ormai vecchio e un discepolo molto giovane, viene addirittura interpretato con una forma di

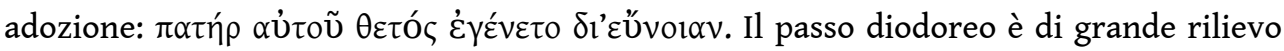
perché mette in rapporto di causa ed effetto l'adesione ai principi della dottrina pitagorea e i successi personali di Epaminonda ${ }^{37}$.

Quella del rapporto filiale è una notizia antica perché si ritrova in uno dei capitoli di Giamblico risalenti ad Aristosseno: «lo chiamò con il nome di padre» $(250)^{38}$.

Porfirio, citando Neante di Cizico (III sec. a.C.) nella sua Vita di Pitagora (55) non si discosta da quanto leggiamo nel suo contemporaneo Giamblico, né per i nomi dei due sopravvissuti, né per il rapporto di discepolato fra Liside ed Epaminonda; però aggiunge, poco più avanti (57) che Liside e Archippo «come tutti quelli che erano stati esiliati, salvarono solo qualche scintilla, oscura e indecifrabile, di quella filosofia», è una affermazione singolare, perché una lunga e diversa tradizione, che ha voluto attribuire a Liside opere scritte, è evidentemente di diverso segno.

Infatti, Liside tarantino e tebano, secondo le complesse manipolazioni della tradizione pitagorica, sarebbe stato l'autore di uno dei tre scritti "di Pitagora", il Physikon ${ }^{39}$ e di due epistole indirizzate ad Ipparco, o meglio Ippaso, come si legge in Diogene Laerzio ${ }^{40}$, per rimproverarlo di aver diffuso per iscritto la dottrina di Pitagora.

Una di queste epistole è tramandata da Giamblico (V.P., 75-78) ${ }^{41}$, il quale l'ha ripresa da Nicomaco di Gerasa, e non sembra conoscere l'altra versione, comunque precedente, conservata negli Epistolographi Graeci ${ }^{42}$; entrambe sono ora datate da Städele ${ }^{43}$ ai secoli III d.C., in base alla presenza di atticismi.

Concludo ritornando alla educazione ricevuta da Epaminonda, alla quale certamente apparteneva la nozione dei dieci principi elencati nella Metafisica di Aristotele $(1,4)$, ed in particolare quello della destra e della sinistra ${ }^{44}$, facilmente applicabile all'uso delle armi $^{45}$, nel quale Epaminonda ebbe tanti successi, e che riporta esplicitamente all'immagine del bivio dell'adolescente Eracle, di cui sopra si è fatta breve memoria, rimandando alla vastissima bibliografia specifica.

\section{BIBLIOGRAFIA}

ALONI Antonio, Il demone di Socrate, traduzione e note, Milano, Adelphi, 1982.

BOYANCÉ Pierre, Le culte des muses chez les philosophes grecs, Paris, De Boccard, 1936 (anastatica 1972). 
BREGLIA Luisa, Polymnis, padre di Epaminonda e la tradizione pitagorica, in Antiquitas. Studi di storia antica in onore di S. Alessandri, Galatina, 2011, pp. 11-15.

BREGLIA Luisa, Ferecide di Siro tra Orfici e Pitagorici, in Tra Orfeo e Pitagora (Atti seminari napoletani 1996-1998), Napoli, Bibliopolis, 2000, pp. 161-194.

BUCKLER John, Epaminondas and Pythagoreanism, «Historia», 1993 (2000), pp. 104-108.

CARLINI Antonio, Sulla composizione della Tabula di Cebete, «SCO», 12, 1963, pp. 164-182.

CONZE Alexander, Die Attischen Grabreliefs, t. IV, Wien, Österreichische Akademie der Wissenschaften, 1914.

CUMONT Franz, Recherches sur le symbolisme funéraire des Romains, Paris, 1942.

DETIENNE Marcel, Sur la démonologie de l'ancien pythagorisme, «Rev. Hist. Rel.», 155, 1959, pp. 17-32.

DETIENNE Marcel, Héraclès héros pythagoricien, «Rev. Hist. Rel.» 158, 1960, pp. 19-53.

DONINI Pierluigi (a cura di), Plutarco. Il demone di Socrate, introduzione, traduzione e commento di P. Donini, Roma, Carocci, 2017.

D'ORLANDO Dario, Il sepolcro di Orfeo. Appunti per l'identificazione di un rituale funerario orficobacchico, «Otium», 2, 2017. Disponibile all'indirizzo: <www.otium.unipg.it/otium/article/view/ 21> (data di accesso: 18 apr. 2018).

GIANGIULIO Maurizio (a cura di), Pitagora. Le opere e le testimonianze, Milano, Mondadori, 2000.

HAFNER Markus, Die Macht der Rede in der Tabula Cebetis, «Hermes», 141, 2013, pp. 65-82.

HERCHER Rudolph, Epistolographoi Ellenikoi, Parisiis, 1873.

HUFFMAN Carl, Philolaos of Croton, Cambridge, Cambridge University Press, 1993.

JOLY Robert, Le tableau de Cébès et la philosophie religieuse, coll. Latomus LXI, Bruxelles, 1963.

LÉVÊQUE Pierre \& VIDAL-NAQUET Pierre, Épaminondas pythagoricien ou le problème tactique de la droite et de la gauche, «Historia», 9, 1960, pp. 294-308.

MELE Antonio, Pitagorismo, Megale Hellàs e Italici, in Da Italia a Italia: le radici di un'identità (Atti LI Convegno di Studi sulla Magna Grecia), Taranto, ISAMG, 2011, pp. 237-278.

MOGGI Mauro \& OSANNA Massimo, Pausania. Guida della Grecia. Libro IX. La Beozia, Mondadori, Milano, 2010.

PETZL Georg, Tituli Asiae Minoris, V, 3, Wien, Österreichische Akademie der Wissenschaften, 2007. PRONTERA Francesco, Gli “ultimi” pitagorici. Contributo per una revisione della tradizione, «Dialoghi di Archeologia», IX-X, 1976-1977, pp. 267-332

PUGLIESE CARRATELLI Giovanni, Le lamine d'oro orfiche, Milano, Adelphi, 2001.

SCHADE Gerson, Rechts und Links im Diskurs des Pythagoreismus, «Hermes», 137, 2009, pp. 118-123. SORDI Marta, Diodori Siculi Bibliothecae liber XVI, Firenze, La Nuova Italia, 1969.

SORDI Marta, Propaganda politica nell'azione di Epaminonda, «CISA», II, 1974, pp. 45-53.

STÄDELE Alfons, Die Briefe des Pythagoras und der Pythagoreer, Meisenheim am Glan, Haim, 1980.

THESLEFF Holger, An Introduction to the Pythagorean Writings of the Hellenistic Period, Ăbo Akademi, 1961.

THESLEFF Holger, The Pythagorean Texts of the Hellenistic Period, Ăbo Akademi, 1965. 
VALLET Georges, Le «stenopos » des Muses à Métaponte, in Mélanges de philosophie, de littérature et d'histoire ancienne offerts à Pierre Boyancé, Publications de l'École française de Rome, 22, 1974, pp. 749-759.

VERNANT Jean-Pierre, Aspects mythiques de la mémoire et du temps, in Mythe et pensée chez les Grecs, Paris, Maspero, 1966, pp. 51-94.

VIDAL-NAQUET Pierre, Le chasseur noir, Paris, Maspero, 1981.

VILLARI Susanna, L'Ercole al bivio di Domenico Beccafumi (1486-1551) e l'Ercole Giraldiano, «Studi Giraldiani», I, 2015, pp. 69-110.

VISCONTI Amedeo, Aristosseno di Taranto. Biografia e formazione spirituale, Napoli, Centre J. Bérard, 1999.

ZELLER Eduard \& MONDOLFO Rodolfo, La filosofia dei Greci, I, 2, Firenze, La Nuova Italia, $1964^{2}$.

ZHMUD Leonid, What is Pythagorean in the pseudo-Pythagorean literature?, «Philologus», c.d.s.

\section{NOTE}

1. Poche righe prima (579D) Polimnide, padre di Epaminonda, annuncia il motivo della visita e l'arrivo dello straniero, che è accompagnato da numerose persone (578E). Vd. Donini (2017). Su Polimnide vd. Breglia (2011).

2. Simmia è il personaggio che nel Fedone (61), a dir di Platone, è stato a Tebe discepolo di Filolao.

3. Il nome di Liside è in tutti e due gli elenchi dei Pitagorici di Giamblico: nel primo (V.P., 104) è nominato tra Aristeo ed Empedocle, come a suggerire la datazione al V sec. a.C., nel secondo (V.P., 256) in un lungo elenco di Tarantini apparentemente in ordine di notorietà, forse risalente ad Aristosseno; Giangiulio (2000, 535 e 545).

4. Nello scolio a Phaed. 61a è Filolao che va a Tebe «per rendere onori funebri» a Liside, probabilmente c'è stato uno scambio di nomi, che si ripete nelle notizie sugli scampati all'incendio, alle quali si farà riferimento qui di seguito, Prontera (1976-1977, 267-332, part. 313, nota 152 e 317 , nota 162 ).

5. I Pitagorici sanno distinguere i vivi dai morti che appaiono in sogno: in un altro passo di Plutarco (de sera numinis vidicta, Mor. 564C) leggiamo che gli eidola dei morti non muovono le palpebre e non producono ombra.

6. Detienne (1959, 17-32, part. 21-24).

7. Platone, Phaed., 62.

8. Giangiulio $(2000,381)$, di cui riporto la traduzione. Vd. ora D'Orlando (2017): pur non ritenendo assimilabili i rituali orfici e quelli pitagorici, è però interessante constatare la prevalenza delle inumazioni anche nelle sepolture orfiche.

9. Vernant (1966, 51-94, part. 69) e Breglia (2000, 161-194).

10. Mele (2011).

11. Fr. 14 Wehrli = Giangiulio $(2000,56)$. Per Aristosseno è fondamentale Visconti (1999).

12. FHG III, p. 169 fr. 6; anche Satiro fr. 10. Giangiulio (2000, 251-252).

13. Vallet (1974, 749-759).

14. Mele (2011, 243, nota 42$)$; Giangiulio $(2000,80)$.

15. Zhmud (c.d.s., 218).

16. Commento Moggi-Osanna (2010, 305-306).

17. Nel libro di Boyancé (1936 [anast. 1972], 133-144), rimane insuperato il capitolo «La mort pythagoricienne» anche se non lo seguo nelle conclusioni sui Dioscuri.

18. Pugliese Carratelli $(2001,66)$; Giangiulio $(2000,9)$. 
19. Giamblico, V.P., 155.

20. Trad. Giangiulio.

21. Fr. 23 Werhli = Giangiulio $(2000,90)$.

22. Se fosse un'incinerazione, avremmo la parte di un contenitore, anziché una stele.

23. Petzl (2007, n. 1895), con bibliografia precedente, mancante, tra gli altri di Carlini (1963, 164-182), con descrizione e fotografia della stele di Alasehir, e di Detienne (1960). Fotografia

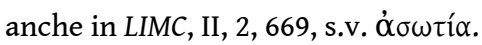

24. Opere e giorni, vv. 289-292.

25. Memorabili, II, 20-34; Cicerone, De officiis, I 31, 117-118 e Filostrato, V. Apollonii, VI, 10.

26. Detienne (1960, 19-53).

27. Cumont (1942, 422-431).

28. La psi, indicante le tre vie, compare su una tavola di marmo rinvenuta sulla strada tra Atene ed Eleusi: Conze (1914, 8, fig. 1743).

29. Joly (1963) e Hafner (2013). La fortuna letteraria e pittorica di tale rappresentazione è oggetto di una bibliografia estesissima, si veda l'importante e recente lavoro di Villari (2015), con un buon repertorio delle fonti classiche.

30. Carlini $(1963,167)$.

31. Mondolfo in Zeller \& Mondolfo $\left(1964^{2}, 426\right)$.

32. Huffman $(1993,3)$.

33. Polibio, II, 39; Prontera (1976-1977); Aloni (1982, 83 e 188); Donini (2017, 179), il quale però non evidenzia il problema.

34. Sopra note 3 e 32 .

35. Diodoro Siculo, XVI, 2; Sordi (1969, 5). Lévêque \& Vidal-Naquet (1960, 294-308).

36. Sordi (1974, 45-54); Lévêque \& Vidal-Naquet (1960) e Vidal-Naquet (1981, 115-121); Buckler (1993, 104-108).

37. Vd. anche Diodoro Siculo, XV, 39,2 e 52,7.

38. Giangiulio $(2000,417)$.

39. Diogene Laerzio, VIII, 6 e Suda, s.v. Pythagoras Samios.

40. Diogene Laerzio, VIII, 42: Ippaso è nome preferibile perché è presente negli elenchi dei Pitagorici, però la tradizione delle lettere porta Ipparco.

41. Giangiulio $(2000,534)$. Anche Diogene Laerzio, VIII, 42.

42. Hercher (1873, 601-603).

43. Städele (1980, 154-159); Thesleff $(1965,111-114)$, che riporta il testo di Hercher. Vd. anche Thesleff (1951, 15 e 113-115) con datazioni alte non più accettabili.

44. Schade $(2009,118-123)$.

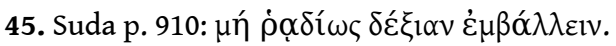

\section{RIASSUNTI}

Liside tarantino è morto a Tebe, dove, essendo stato maestro di Epaminonda, ha avuto una sepoltura giusta per i canoni pitagorici; la notizia viene confrontata con le poche altre informazioni su sepolture di Pitagorici. 
Lysis de Tarente est mort à Thèbes où, ayant été maître d'Épaminondas, il reçut une sépulture adéquate selon les normes pythagoriciennes; cette notice est confrontée avec les rares informations de la tradition sur les sépultures de Pythagoriciens.

Lysis of Taras died in Thebes where, as he was Epaminondas' teacher, he was appropriately buried according to Pythagorean standards; this information is compared with the few traditional data about the graves of some of Pythagoras's disciples.

\section{INDICE}

Mots-clés : Lysis, Épaminondas, sépulture, pythagorisme

Parole chiave : Liside, Epaminonda, sepoltura, pitagorismo

Keywords : Lysis, Epaminondas, burial, Pythagorism

\section{AUTORE \\ FEDERICA CORDANO}

Università degli Studi di Milano.

Professeur émérite d'histoire grecque, Federica Cordano a enseigné aux universités de Rome "La Sapienza ", Macerata et Milan (Statale). Elle dirige la revue Aristonothos et les cahiers d'Aristonothos avec Giovanna Bagnasco. Elle est membre du Deutsches Istorisches Institut de Rome. Ses domaines d'études privilégiés sont la Sicile et l'histoire des cités mégariennes du Bosphore et de la mer Noire. 